\title{
Temperature dependent recombination dynamics in InP/ZnS colloidal nanocrystals
}

\author{
Shirazi, Roza; Kopylov, Oleksii; Kovács, András; Kardynal, Beata
}

Published in:

Applied Physics Letters

Link to article, DOI:

$10.1063 / 1.4749276$

Publication date:

2012

Document Version

Publisher's PDF, also known as Version of record

Link back to DTU Orbit

Citation (APA):

Shirazi, R., Kopylov, O., Kovács, A., \& Kardynal, B. (2012). Temperature dependent recombination dynamics in InP/ZnS colloidal nanocrystals. Applied Physics Letters, 101(9), 091910. https://doi.org/10.1063/1.4749276

\section{General rights}

Copyright and moral rights for the publications made accessible in the public portal are retained by the authors and/or other copyright owners and it is a condition of accessing publications that users recognise and abide by the legal requirements associated with these rights.

- Users may download and print one copy of any publication from the public portal for the purpose of private study or research.

- You may not further distribute the material or use it for any profit-making activity or commercial gain

- You may freely distribute the URL identifying the publication in the public portal 


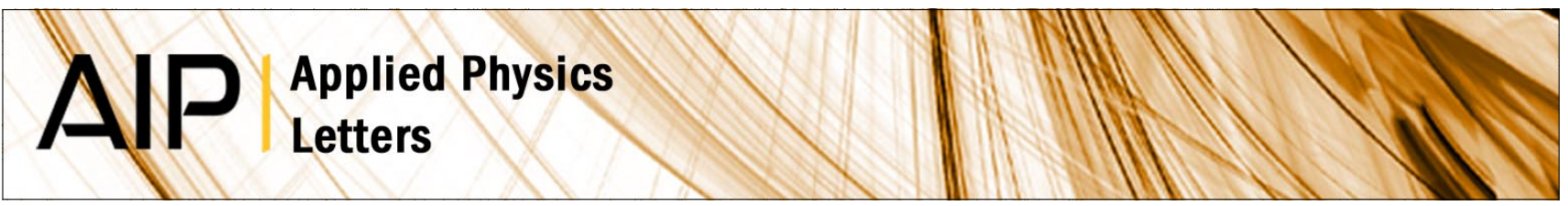

\section{Temperature dependent recombination dynamics in InP/ZnS colloidal nanocrystals}

R. Shirazi, O. Kopylov, A. Kovacs, and B. E. Kardynał

Citation: Appl. Phys. Lett. 101, 091910 (2012); doi: 10.1063/1.4749276

View online: http://dx.doi.org/10.1063/1.4749276

View Table of Contents: http://apl.aip.org/resource/1/APPLAB/v101/i9

Published by the American Institute of Physics.

\section{Related Articles}

Importance of excitonic effects and the question of internal electric fields in stacking faults and crystal phase quantum discs: The model-case of GaN

J. Appl. Phys. 112, 053512 (2012)

Enhancement of coherent energy transport by disorder and temperature in light harvesting processes JCP: BioChem. Phys. 6, 09B603 (2012)

Enhancement of coherent energy transport by disorder and temperature in light harvesting processes

J. Chem. Phys. 137, 094107 (2012)

Dynamics of a Holstein polaron with off-diagonal coupling

J. Chem. Phys. 137, 084113 (2012)

On the mechanisms of energy transfer between quantum well and quantum dashes

J. Appl. Phys. 112, 033520 (2012)

\section{Additional information on Appl. Phys. Lett.}

Journal Homepage: http://apl.aip.org/

Journal Information: http://apl.aip.org/about/about_the_journal

Top downloads: http://apl.aip.org/features/most_downloaded

Information for Authors: http://apl.aip.org/authors

\section{ADVERTISEMENT}

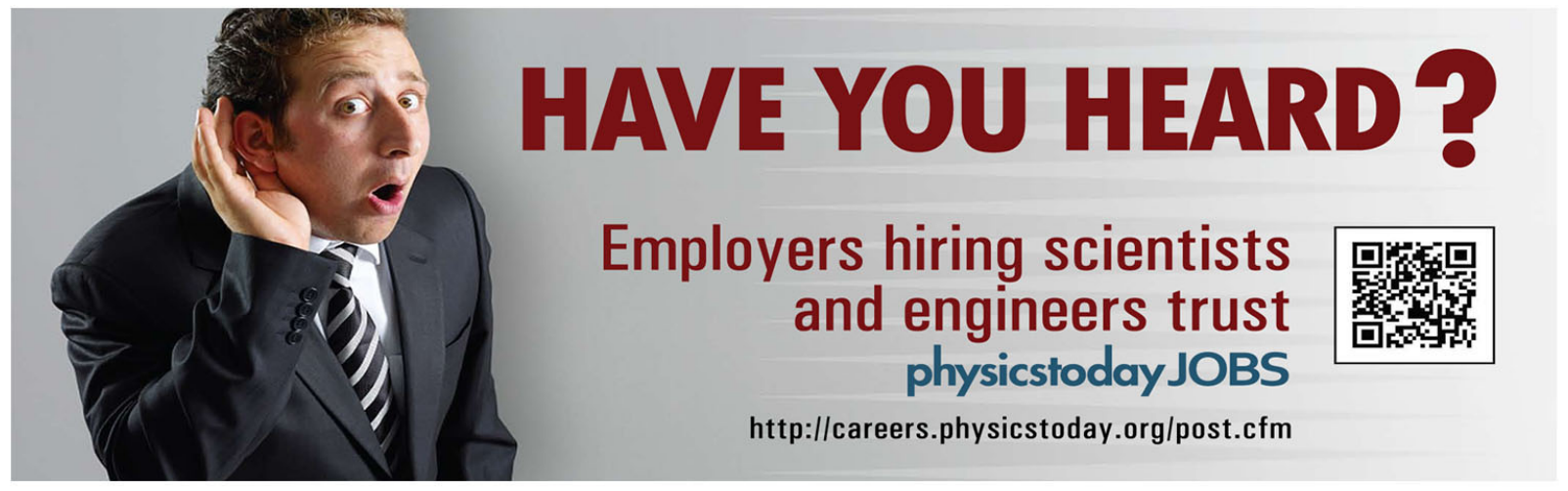




\title{
Temperature dependent recombination dynamics in InP/ZnS colloidal nanocrystals
}

\author{
R. Shirazi, ${ }^{1}$ O. Kopylov, ${ }^{1}$ A. Kovacs, ${ }^{2}$ and B. E. Kardynat ${ }^{1,3}$ \\ ${ }^{1}$ DTU Fotonik, Technical University of Denmark, Kgs Lyngby, Denmark \\ ${ }^{2} C E N$, Technical University of Denmark, Kgs Lyngby, Denmark \\ ${ }^{3}$ PGI-9, Forschungszentrum Jülich, JARA FIT, Jülich, Germany
}

(Received 9 June 2012; accepted 17 August 2012; published online 30 August 2012)

\begin{abstract}
In this letter, we investigate exciton recombination in $\mathrm{InP} / \mathrm{ZnS}$ core-shell colloidal nanocrystals over a wide temperature range. Over the entire range between room temperature and liquid helium temperature, multi-exponential exciton decay curves are observed and well explained by the presence of bright and dark exciton states, as well as defect states. Two different types of defect are present: one located at the core-shell interface and the other on the surface of the nanocrystal. Based on the temperature dependent contributions of all four states to the total photoluminescence signal, we estimate that the four states are distributed within a $20 \mathrm{meV}$ energy band in nanocrystals that emit at $1.82 \mathrm{eV}$. (C) 2012 American Institute of Physics. [http://dx.doi.org/10.1063/1.4749276]
\end{abstract}

Colloidal nanocrystals (NCs) have been a subject of intense studies due to their potential applications in photonic devices. ${ }^{1}$ The simple and cheap fabrication makes them particularly attractive in applications such as medical diagnostics, solid state lighting, or solar cells ${ }^{2}$ especially when made of benign materials such as InP. Optical studies of the colloidal nanocrystals have, however, revealed a complex carrier dynamics, which needs to be understood before the full benefit of the nanocrystals can be achieved. A finite size as well as the quantisation of states in small nanocrystals modifies their band structure compared with bulk materials, often leading to different optical transitions compared with the ones familiar from the latter. ${ }^{3}$ Radiative recombination of photo-excited carriers competes in NCs with trapping on surface states ${ }^{4}$ and with Auger recombination. ${ }^{5}$ Reduction of the parasitic recombination channels is of paramount importance for implementation of nanocrystals in devices and it requires detailed understanding of the carrier dynamics.

Characterisation of the nanocrystals and methods to interpret the data needed to undergo rapid development to keep pace with their synthesis. Time resolved photoluminescence (TRPL) has often been used to study the carrier dynamics in the nanocrystals. TRPL decay curves have been usually fitted as double exponential function with two time constants of the order of a few and a few tens of nanoseconds. However, due to often difficult assignment of decay rates to recombination processes in ensemble measurements, there is a disagreement in the literature over the contribution of core excitons and defect states to the measured signal. Sometimes the longer time constant is interpreted as a radiative recombination of excitons, ${ }^{6}$ while in other reports it is thought to be related to recombination via surface states. ${ }^{7}$

In this letter, we use temperature dependence of time resolved photoluminescence of $\mathrm{InP} / \mathrm{ZnS}$ colloidal nanocrystals combined with multi-exponential fitting in order to identify the processes responsible for carrier recombination.

$\mathrm{InP} / \mathrm{ZnS}$ nanocrystals ${ }^{8}$ coated with oleylamine were purchased from NN-Labs. From energy-dispersive x-ray spectroscopy, the average InP core radius to $\mathrm{ZnS}$ shell thickness was $0.87: 0.13$. Statistics of the nanocrystals sizes from high resolution transmission electron micrographs (HRTEM, not shown here) revealed that the average size of the InP nanocrystals was $3.5 \mathrm{~nm}$ with a full width at a half maximum (FWHM) of $1.3 \mathrm{~nm}$ as shown in the inset to Figure 1. The nanocrystals were drop casted on quartz substrates and mounted on a cold finger of the helium cooled cryostat for the cryogenic measurements. Excess ligands (ligands not bound to the nanocrystals) ensured that the nanocrystals were kept on average $10 \mathrm{~nm}$ apart. The photoluminescence spectrum from the nanocrystals measured on the quartz substrate at room temperature had a maximum at $642 \mathrm{~nm}$, consistent with the nanocrystals size ${ }^{9}$ and a FWHM of $57 \mathrm{~nm}$, compared to the estimated from the size distribution $(88 \mathrm{~nm})$ with the discrepancy most likely related to the distribution of the shell thickness as well as the core thickness. Upon cooling to $10 \mathrm{~K}$, the emission wavelength shifted to $632 \mathrm{~nm}$ reflecting increased bandgap while the FWHM decreased to $50 \mathrm{~nm}$, as expected from eliminating phonon broadening. ${ }^{10}$ A photoluminescence (PL) spectrum at an intermediate temperature of $140 \mathrm{~K}$ is shown in Figure 1.

For time resolved measurements, samples were excited with a pulsed diode laser emitting at the wavelength of

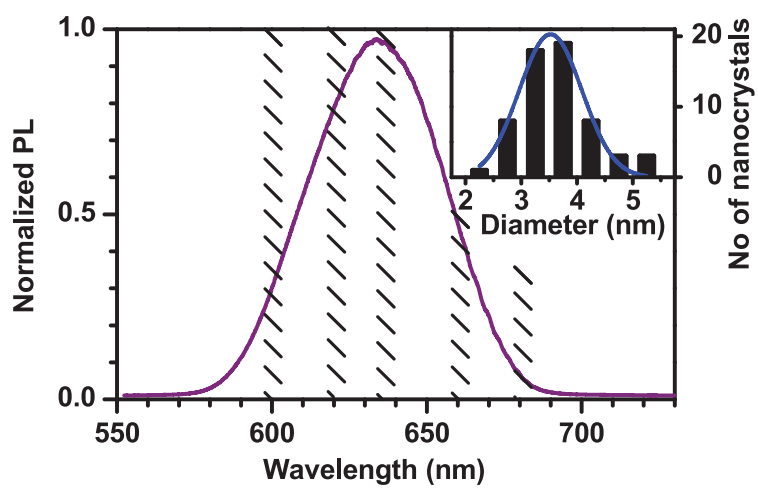

FIG. 1. Photoluminescence spectrum at $140 \mathrm{~K}$. Shaded areas mark the parts of the spectrum selected for time resolved measurements. Inset shows the size distribution of the NCs size determined from HRTEM image. 
$405 \mathrm{~nm}$, which is above the bandgap of InP core but below the bandgap of $\mathrm{ZnS}$ shell. The laser excitation consisted of a train of $70 \mathrm{ps}$ long light pulses, with the repetition rate of 2.5 MHz. Laser power incident on the sample was set to be $15.6 \mu \mathrm{W}$, which corresponds to the power density of $138 \mathrm{Wcm}^{-2}$. From calculations of the light absorption by the nanocrystals, ${ }^{11}$ we expect an average generation of about 0.6 excitons per nanocrystal per laser pulse. Due to the Poissonian distribution of absorbed light, $10 \%$ of NCs were thus excited with more than one exciton, $33 \%$ of NCs were excited with one exciton and 54\% were not excited. Light emitted by the nanocrystals was focused on an input slit of a monochromator; a narrow band $(7 \mathrm{~nm})$ of the spectrum centred at the selected wavelengths (see shaded areas in Figure 1) was isolated with an output slit and focused on a Si single photon avalanche photodiode (APD). Decay curves at these particular emission wavelengths were measured using time resolved single photon counting. Time resolution of the system, determined by the laser diode pulse width and the response time of the APD, was $200 \mathrm{ps,} \mathrm{while} \mathrm{the} \mathrm{photolumi-}$ nescence decay curves were recorded over one period of the laser signal, $400 \mathrm{~ns}$.

Figure 2(a) shows exciton decay curve measured at $280 \mathrm{~K}$. The curve is non-exponential but it can be fitted with three exponential components: $y(t) / y(0)=\sum_{i=1}^{3} A_{i} e^{-k_{i} t}$, where $\sum_{i=1}^{3} A_{i}=1$, with rates: $k_{1}=0.25 \mathrm{~ns}^{-1}, k_{2}=0.08 \mathrm{~ns}^{-1}$,
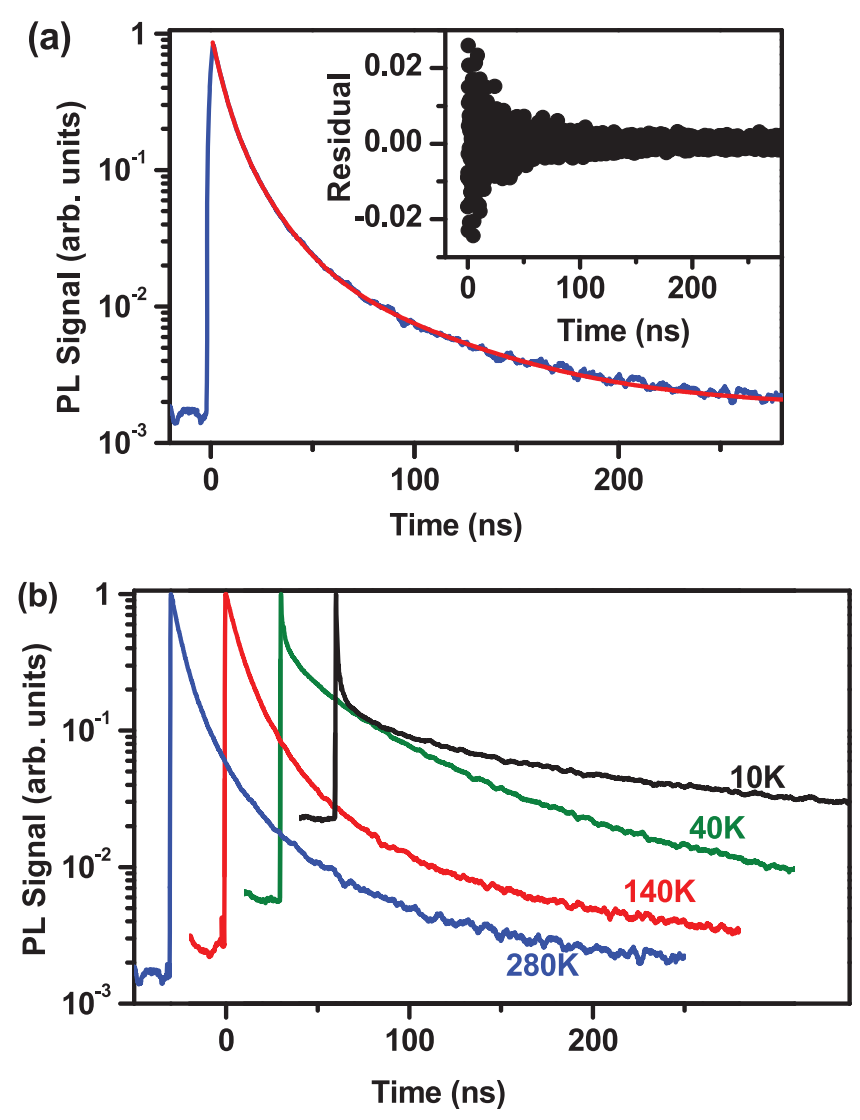

FIG. 2. Time resolved photoluminescence following a pulse of laser light at time zero measured at $637 \mathrm{~nm}$ (a) at $280 \mathrm{~K}$ together with the fitted curve (red line) and (b) at different temperatures. Due to periodic excitation with $400 \mathrm{~ns}$ period, the signal level at $\mathrm{t}<0$ indicates that not all excitons decayed before the pulse of laser at time $t=0$. Inset to figure (a) shows residuals for the fitting. and $k_{3}=0.02 \mathrm{~ns}^{-1}$ and amplitudes: $A_{1}=0.675, A_{2}=0.299$, and $A_{3}=0.025$. The plot of residuals in the figure inset confirms the goodness of the fit. Most commonly used fitting functions such as log-normal distribution of lifetimes and stretched exponential were also tested but did not give better results than the three exponential functions.

In our excitation scheme, only a small fraction of nanocrystals is excited with more than one exciton and since the measured signal did not change even when the excitation power was reduced by factor of 10 (with the resulting probability of multiple excitons generation of $0.17 \%$ ), we can assume that the multiexciton effects can be neglected in our analysis. In this case as long as the excitons decay within one period of the laser excitation, each measured photon originates from a different nanocrystal as a result of different decay processes.

The radiative recombination rate of excitons in an InP nanocrystal emitting at $637 \mathrm{~nm}$ can be estimated as $0.2 \mathrm{~ns}^{-1}$, if the electron-hole wavefunctions have overlap of unity. ${ }^{11}$ However, there is a finite energy splitting between dark and bright exciton states due to the exchange coupling ${ }^{12}$ with the 5 -fold degenerate dark exciton being the lowest energy state and the 3-fold degeneracy of the bright exciton in InP nanocrystals. Since the expected energy splitting is much lower than the thermal energy at room temperature, the presence of dark state leads to the reduced recombination rate of $0.075 \mathrm{~ns}^{-1}$. Although this rate is comparable to $k_{2}$, it is not sufficient to assign it to the core exciton due to unknown non-radiative recombination rate.

In order to differentiate between the possible decay processes, we measured temperature dependence of the TRPL. The evolution of the photoluminescence decay with temperature measured for $637 \mathrm{~nm}$ emission on an ensemble of nanocrystals between room temperature and $10 \mathrm{~K}$ is shown in Figure 2(b). In the range of temperatures between room temperature and $140 \mathrm{~K}$, the curves did not change much. Upon further cooling, the photoluminescence dynamics transformed drastically. The slow decay component became more pronounced leading to incomplete recombination during one period of laser excitation, seen as an increasing PL signal level just before arrival of a laser pulse at lower temperatures (e.g., PL of 0.02 of initial value at $10 \mathrm{~K}$ in Figure 2(b)). This in turn leads to the appearance of Auger recombination with a very fast decay rate, $k_{4}\left(2 \mathrm{~ns}^{-1}\right.$, limited by the temporal resolution of the experiment), which gradually increased in strength as the temperature was reduced. Auger recombination has been commonly observed in nanocrystals populated with excess charges or multi-excitons ${ }^{13}$ and it is considered a source of major optical losses in nanocrystals.

To better visualize the changes, we plot in Figure 3 all the decay rates as well as more familiar average decay rate defined as $k_{a v}=\left(\sum_{i=1}^{n} A_{i} k_{i}^{-1}\right) /\left(\sum_{i=1}^{n} A_{i} k_{i}^{-2}\right)$, where $n=3$ or 4 depending on the number of decay components present at a given temperature. The values of decay rates $k_{1}$ and $k_{2}$ are only weakly changing with temperature, while $k_{3}$ undergoes very strong oscillation around $50 \mathrm{~K}$. This latter behaviour is very characteristic of traps in resonance with the core excitonic levels ${ }^{4}$ which combined with low value of $k_{4}$ points to the external surface of the nanocrystal as the location of the traps. 


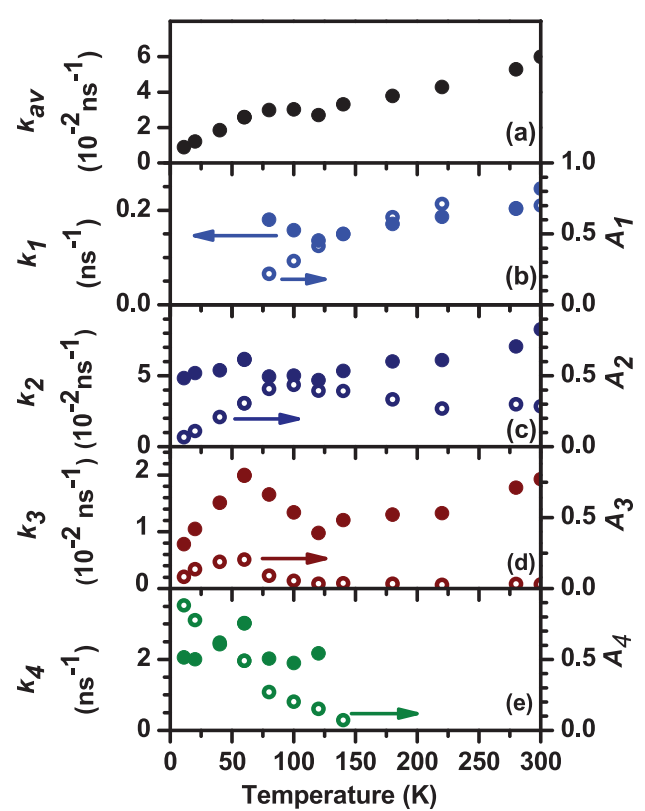

FIG. 3. Evolution of average decay constant, $k_{a v}$ (a) and four single exponential components: $k_{1}$ (b), $k_{2}$ (c), $k_{3}$ (d), and $k_{4}$ (e) with temperature. Right hand scale indicates the pre-exponential factor, $A_{i}$, in the fitting.

An amplitude of $k_{1}$ component of the decay curves decreases until at about $70 \mathrm{~K}$ and it becomes too small to be extracted by fitting. A gradual reduction of this component is associated with an increase of amplitudes of $k_{2}$ and $k_{3}$ components. Upon further cooling, the amplitude of the $k_{2}$ component starts falling while $k_{3}$ component continues to grow until finally it also decreases below $30 \mathrm{~K}$, while Auger recombination $\left(k_{4}\right)$ steadily increases down to liquid helium temperature. It is worth noting that although the amplitude of the Auger recombination, $A_{4}$, is very high compared with other components, its contribution to the total PL signal $\left(=A_{4} / k_{4}\right)$ remains very small (less than $\left.10 \%\right)$.

The evolution of $k_{1}, k_{2}$, and $k_{3}$ components can be explained as originating from a system of coupled four-level system with different energies (see Figure 4), with the lowest dark exciton energy state and three states, responsible for the $k_{1}, k_{2}$, and $k_{3}$ components in the order of decreasing energy above the dark exciton state. The energy separation of the state responsible for $k_{1}$ component can be estimated as $12 \mathrm{meV}$, as it is quenched at about $50 \mathrm{~K}$. It is not possible to perform similar estimation of the energy of the states responsible for $k_{2}$ and $k_{3}$ components due to strong presence of $k_{4}$ component in the temperature range, in which they are being quenched.

The same measurements and analysis performed for the emission centred at other wavelengths between $600 \mathrm{~nm}$ and $660 \mathrm{~nm}$ (see Figure 1) yielded qualitatively similar results.

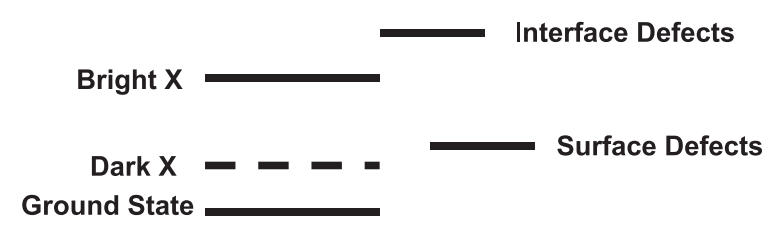

FIG. 4. Diagram of the exciton states present in the InP/ZnS nanocrystals.
There were quantitative differences in the temperatures at which the different components of PL were quenched, the temperature at which the Auger component $\left(k_{4}\right)$ appeared and amplitudes of various components.

An analysis of the emission at these different wavelengths yielded a dependence of the energetic position of the state responsible for the decay rate $k_{1}$ as a function of emission energy, shown in Figure 5. As the exciton energy increases, the separation between the dark exciton and the analysed state decreases. Such dependence clearly points to the defect being the origin of the $k_{1}$ component in agreement with previous studies of InP nanocrystals. ${ }^{14}$ Note that at the emission energy of $2.08 \mathrm{eV}$, the $k_{1}$ and $k_{2}$ components are quenched in the same temperature range indicating that this state is very close in energy to the $k_{2}$ component. Similar trend-lower energy separation from the dark exciton in smaller nanocrystals-is observed for the $k_{3}$ component, leaving $k_{2}$ as originating from the core bright exciton. Defects located near the core of the nanocrystal (on the interface between the core and the shell) are the most likely origin of $k_{1}$ component as $k_{1}$ is fast and the ratio of $A_{1}: A_{2}$ is wavelength independent at room temperature (which means that the states are in thermal equilibrium). Note that the measured at room temperature core exciton decay rate, $k_{2}=0.08 \mathrm{~ns}^{-1}$ is higher than the calculated value for radiative recombination rate of $0.054 \mathrm{~ns}^{-1}$ indicating the presence of non-radiative recombination.

The picture of the energy states of the InP/ZnS nanocrystals studied here is consistent with the one determined for etched InP nanocrystals passivated with organic ligands. ${ }^{14,15}$ It is not surprising that in both types of NCs, the splitting between the bright and dark excitons is similar as this is an intrinsic property of the InP nanocrystals. ${ }^{12}$ The dominant at room temperature parasitic recombination channel in the InP/ZnS is also very similar to that of the passivated InP nanocrystals, ${ }^{15}$ proving the protective role of $\mathrm{ZnS}$ shell. The origin of this recombination channel is not clear. Although thin shell may not be continuous and so InP surface may be partly exposed, numerically predicted positions of In and P dangling bonds inside the InP bandgap ${ }^{16}$ cannot explain the results.

The one-pot fabrication method, used for the synthesis of the nanocrystals under study, relies on forming $\mathrm{ZnS}$ shells

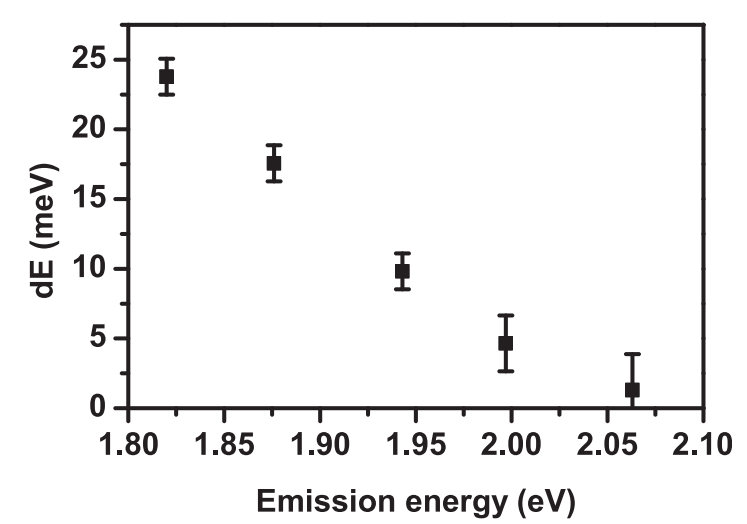

FIG. 5. Estimated splitting between defect state (at higher energy) and lowest exciton energy (dark exciton) as a function of the energy of emission (nanocrystal size). 
by adding the $\mathrm{Zn}$ and $\mathrm{S}$ precursors to the solution of In and $\mathrm{P}$ precursors after InP cores have been formed. It has been suggested $^{8}$ and later observed ${ }^{17}$ that the shell grown under such conditions is likely to be an alloy of $\mathrm{InP}$ and $\mathrm{ZnS}$, resulting in gradual potential transition across the interface between the core and shell rather than abrupt InP/ZnS junction. Such an interface may be vulnerable to the formation of defects responsible for non-radiative recombination. ${ }^{18}$

The onset of Auger recombination $\left(k_{4}\right)$ shifts to higher temperatures and its amplitude increases for shorter wavelength emission. Relying on the transfer of energy to a third particle (electron or hole), Auger recombination is only possible in nanocrystals which are charged or occupied with more than one exciton. The second case, an occupation of the nanocrystals with more than one exciton is in our experiments caused by the carriers freeze out on the dark exciton states. It is clear, however, that higher levels of excitation at room temperature would also lead to Auger recombination quenching of radiative recombination. This demonstrates the detrimental effect of the presence of slow dark exciton states on photon emission. ${ }^{13}$

In conclusion, we have measured temperature dependent radiative recombination of excitons in an ensemble of InP/ZnS nanocrystals. The analysis of the decay dynamics has indicated that initially generated exciton population decays to four energy levels, which we have assigned in the order of increasing energy as dark exciton, surface defect, bright exciton, and core-shell interface defect states. The interface defect states in the smallest nanocrystals, emitting at $600 \mathrm{~nm}$, are very close in energy to the bright exciton state while the surface states are close in energy to the dark excitons. Below about $140 \mathrm{~K}$, long-lived dark excitons lead to strong Auger recombination. The results show that while a thin $\mathrm{ZnS}$ shell protects the surface of InP from oxidation, it does not change the recombination dynamics in the nanocrystals and so engineering of the energy level splitting and defect elimination remain crucial factors in the design of highly emissive nanocrystals. ${ }^{19}$

${ }^{1}$ V. Klimov, J. Phys. Chem. B 110, 16827 (2006).

${ }^{2}$ D. V. Talapin, J.-S. Lee, M. V. Kovalenko, and E. V. Shevchenko, Chem. Rev. 110, 389 (2010), and references therein.

${ }^{3}$ Al. L. Efros, M. Rosen, M. Kuno, M. Nirmal, D. J. Norris, and M. Bawendi, Phys. Rev. B 54, 4843 (1996).

${ }^{4}$ M. Jones, S. S. Lo, and G. D. Scholes, J. Phys. Chem. C 113, 18632 (2009).

${ }^{5}$ F. Garcia-Santamarı, Y. Chen, J. Vela, R. D. Schaller, J. A. Hollingsworth, and V. I. Klimov, Nano Lett. 9, 3482 (2009).

${ }^{6}$ U. Thuy, P. Thuy, N. Liem, L. Li, and P. Reiss, Appl. Phys. Lett. 96, 073102 (2010).

${ }^{7}$ M. Kim, J. Chung, M. Lee, S. Lee, and D.-J. Jang, J. Colloid Interface Sci. 350, 5 (2010).

${ }^{8}$ R. Xie, D. Battaglia, and X. Peng, J. Am. Chem. Soc. 129, 15432 (2007).

${ }^{9}$ H. Yu, J. Li, R. A. Loomis, L.-W. Wang, and W. Buhro, Nature Mater. 2, 517-520 (2003).

${ }^{10}$ A. Narayanaswamy, L. F. Feiner, A. Meijerink, and P. J. van der Zaag, ACS Nano, 3, 2539-2546 (2009).

${ }^{11}$ I. Moreels, K. Lambert, D. De Muynck, F. Vanhaecke, D. Poelman, J. C. Martins, G. Allan, and Z. Hens, Chem. Mater. 19, 6101-6106 (2007).

${ }^{12}$ A. Franceschetti, H. Fu, L. W. Wang, and A. Zunger, Phys. Rev. B 60, 1819 (1999)

${ }^{13}$ J. A. McGuire, J. Joo, J. M. Pietryga, R. D. Schaller, and V. I. Klimov, Acc. Chem. Res. 41, 1810 (2008).

${ }^{14}$ S. H. Kim, R. H. Wolters, and J. R. Heath, J. Chem. Phys. 105, 7957 (1996).

${ }^{15}$ O. I. Mićić, H. M. Cheong, H. Fu, A. Zunger, J. R. Sprague, A. Mascarenhas, and A. J. Nozik, J. Phys. Chem. 101, 4904 (1997).

${ }^{16}$ H. Fu and A. Zunger, Phys. Rev. B 56, 1496 (1997).

${ }^{17}$ K. Huang, R. Demadrille, M. G. Silly, F. Sirotti, P. Reiss, and O. Renault, ACS Nano 4, 4799 (2010).

${ }^{18}$ P. T. Thuy, U. T. D. Thuy, T. T. K. Chi, L. Q. Phuong, N. Q. Liem, L. Li, and P. Reiss, J. Phys.: Conf. Ser. 187, 012014 (2009).

${ }^{19}$ S. Brovelli, R. D. Schaller, S. A. Crooker, F. García-Santamaría, Y. Chen, R. Viswanatha, J. A. Hollingsworth, H. Htoon, and V. I. Klimov, Nat. Commun. 2, 280 (2011). 